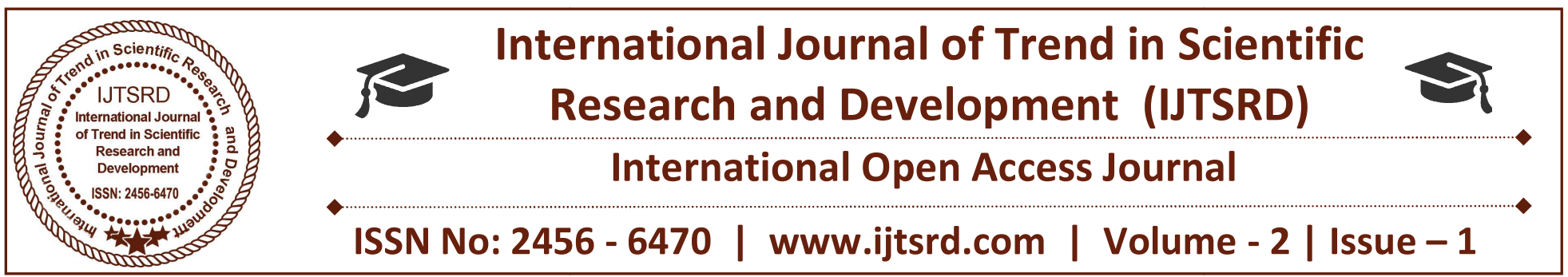

\title{
A Study of an Important Role of Technology in Logistics Sector
}

\author{
M. Indumathi \\ MBA $2^{\text {nd }}$ Year, Saveetha \\ School Of Management, Chennai
}

\author{
Mr. M. Siva \\ MBA, MHRM, MFT, Msc(Uk), NET, \\ Assistant Professor, Saveetha School of \\ Management, Chennai
}

\begin{abstract}
The evolution of business has rapidly increased over the last 25 years. The speed of technological advances has impacted all aspect of business, including the entire supply chain and logistics sectors like manufacturing, warehousing, transportation etc. Every business has looked to capitalize on technology to increase productivity and leveraging that into higher profit margins. With the Internet of Things, the connectivity has helped many businesses shine lights in places that have always been. Companies invest a lot of time, effort, and money in developing technology systems to power the logistics and supply chain.
\end{abstract}

\section{INTRODUCTION}

The logistics management defines logistics as "that part of supply chain process that plans, implements, and controls the effective and efficient forward and reverse flow and storage of goods, services, and related information between the point of origin and the point of consumption in order to meet customer requirement". This should be right product, at the right place, in right time, and in right condition. However supply chain consists of all stages that are required to satisfy the customer request. It starts from supplier passes through manufacturer, distribution, and retailer and finally reaches the customer.

The emerging new technologies are creating strategic opportunities for the organizations to build competitive advantages in various functional areas of management including logistics and supply chain management. However the degree of success depends on the selection of the right technology for the right application. In logistics, information, communication and automation technologies has substantially increased speed of identification, data gathering, processing, analysis and transmission, with high level of accuracy and reliability. Technology is a means to enhance business competitiveness and performance. It plays a major role in success of supply chain by enhancing the overall effectiveness and efficiency of the logistics system. In logistics sector many advanced new technologies using in developed countries but in India it is effective but not that much efficiency in technologies

This is due to liberalization of the Indian economy the competitive pressure is building up and the only option to face the competition is move on to technological operations.

Keywords: logistics efficiency, supply chain management, technology

\section{OBJECTIVES}

$>$ This study is to find out the important role of technology in logistics sector.

To determine the various technologies used in logistic and supply chain.

\section{Bar coding}

Bar coding is a sequence of parallel lines of different thickness with spaces in between. These bars are 
nothing but the items of information in the codified form, which can be read with the help of a scanner.

\section{Radio Frequency Identification (RFID)}

RFID is an Automatic Identification and Data Capture (AIDC) technology. RFID first appeared in tracking and access applications during 1980. RFID-based systems allows for non-contact reading and are effective in manufacturing and other hostile environment where bar codes could not survive. These are used as an alternative to Barcodes to communicate the inventory data to the reader via radio waves. RFID wirelessly exchanges information between a tagged object and a reader.

\section{Electronic Data Interchange (EDI)}

EDI technology is used for transfer of business documents from one computer to other computer. With EDI the business documents such as invoices, cheques, and chellans are sent electronically from one organization to another. In fact EDI is a drive towards paperless document transfer or transactions. The difference between the email message and EDI message is that, E- mail is composed and interpreted manually, while EDI message is composed using one software and interpreted by other software. E-mail data is not structured while EDI data or message is structured.EDI message has legal standing in the court of law.

\section{Geographical positioning System (GPS)}

The GPS is more accurate system used in developed countries wherein a vehicle could be traced accurately with the help of Geo Stationary Satellites to the accuracy of one meter in terms of latitude and longitude. Once the position of vehicle is known, be transmitted to consigner or consignee through the transmission network i.e. mobile phones or internet.

\section{Web Based Tracking}

Logistics service providers operating in India are extending the services of web- based tracking of consignments to their clients. AFL, Fed-Ex, Blue Dart and others are providing the status report of the consignment to their clients. The clients can download this report by connecting through the Internet. This information helps in planning the dispatch schedule and also making follow up with clients for payment collections.

\section{Automated Guided Vehicle System (AGVS)}

The system makes use of magnetic or optical guidance system. The magnetic system uses energized wire laid on the warehouse floor for guiding the material handling equipment. In AGVS operator is eliminated. The new generation AVGS are guided with video and do not follow the fixed path. AGVS can perform all the material handling operation without any human involvement. Robot coupled with AGVS is used to pick up exact material requirement for a customer order.

\section{Enterprise Resource Planning (ERP)}

ERP is integrated software, encompassing all the business operations and bring about significant change in the way people work. ERP is a business solution that addresses to certain identified business issues. ERP is very expensive and complex exercise which require sufficient amount of planning. In India major ERP in use is SAP, Oracle which has been developed by foreign companies to suit the business environment prevailing in those countries. However, some Indian companies like Ramco Systems developed ERP to suit Indian business environment

ERP helps in optimization of supply chain management and develop competitiveness by ensuring the following advantages...

- Quicker response to customer requirement.

- Reduction in inventory costs.

- Improvement in service levels- internal and external.

- Improvement in inventory turnover rate

- Reduction in logistics cost.

Some ERP like.

- Warehouse management system (WMS)

- Transport management system (TMS)

- Material requirement planning (MRP)

- Distribution resource planning (DRP)

- Computer aided dispatch (CAD)

- Systematic analysis process (SAP)

- Automated storage and retrieval system (ASRS)

Internet of things (IOT) is the network of physical objects or things embedded with the electronics, software, sensors, and network connectivity, which enables these objects to collect and exchange data. IOT allows objects to be sensed and controlled 
remotely across existing network infrastructure, creating opportunities for more direct integration between the physical world and computer based system, and resulting in efficiency, accuracy and economic benefits.

For 2020, our Logistics Product Experts and Trend Analyst are looking forward to second generation of IOT, Big Data and Cloud based products. With market focusing on providing various delivery and pickup options, it has become essential for the Logistics Service Providers to ensure maximum utilization of their assets with tight control on wastage DRONES an unmanned aerial vehicles, commonly known as a drone, is an aircraft without a human pilot. This may operates either under the remote control by the human operator or autonomously by on-board computers this plan is on process to implement in the logistics operation. Currently amazon.com is testing on these drones for efficient of door delivery

\section{LITERATURE REVIEWS}

In this article, Logistics and supply chain management refers to the practices and processes aiming for effective and efficient flow of materials and information between a company and its immediate suppliers and customers.

As for technology, when discussing the use of technology in logistics, we refer to the use of interorganizational systems that are used for information sharing and processing across organizational boundaries.

Literature deals with the use of IT and technology in logistics and supply chain. Gunasekaran and Ngai (2004), for example, have included over a hundred journal articles in their recent literature survey addressing the use of information systems in logistics and supply chain integration and management.

There are some few classification frameworks that describe how Technology is used for logistics and supply chain. Bagchi and Skjoett-Larsen (2002) examine the role of IT and organizational integration in logistics integration and propose and technology integration path from low through medium to high integration. The mistocleousetal.(2004), in turn, use the scale of low, medium, too high to describe the integrativeness of different technology alternatives. The focus of the studies being on assessing the levels of supply chain integration, they do not describe how IT is actually used for SCM purposes.

Meanwhile, in their research, Kauremaa et al. (2004) have focused on the ways companies use technology in logistics and supply chain management and choose to classify the use of technology in logistics and supply chain management to transaction execution and information sharing. Based on the rarity of using technology for information sharing among their sample of companies studied, they hypothesize that the drivers and prerequisites of using IT in transaction execution and in information sharing differ, and that the benefits often technology in transaction execution are easier to quantify. Furthermore, they suppose that companies use tech for transaction execution in situations with high transaction volumes and stable business relationships, while IT is used for information sharing especially in environments with demand uncertainty and frequent product introductions. The most severe limitation of this study is that the drivers of using IT for transaction execution and information sharing are hypothesized and cannot bewail dated by their research data.

\section{CONCLUSION}

Technology is a vehicle to lead the competitiveness and keep updated with market and Enhancing the overall effectiveness to make more efficiency of logistics system that efficiency will possible only by the technology and choosing the right technology for various logistics activities or sub-processes is very crucial to any business to gain competitive advantage in today's competitive market.

\section{REFERENCE}

1. Ala-Risku, T., Kärkkäinen, M. and Holmström, J. (2003), "Evaluating the applicability of mergein-transit: A step by step process for supply chain managers", International Journal of Logistics Management, Vol. 14, No. 2, pp. 67-81.

2. Auramo, J., Kauremaa, J. and Tanskanen, K. (2005),"Benefits of technology in supply chain management - an explorative study of progressive companies", International Journal of Physical Distribution \& Logistics Management, Vol. 35, No. 2, pp. 82-100.

3. Bagchi, P.K. and Skjoett-Larsen, T. (2002), "Integration of information technology and organizations in a supply chain", The 
International Journal of Logistics Management, Vol. 14, No. 1, pp. 89-108.

4. Burgess, R. (1998), "Avoiding supply chain management failure: lessons from business process re-engineering", International Journal of Logistics Management, Vol. 9, No. 1, pp. 15-23.

5. Cachon, G.P. and Fisher, M.L. (2000), "Supply chain inventory management and the value of shared information", Management Science, Vol. 46, No. 8, pp. 1032-1048. Christopher, M. (1992), "Logistics and supply chain management", Pitman publishing, London.

6. Christopher Martin, Logistics And Supply Chain Management, Pitman Publishing CoLondon,2001.

7. Coye J.J, Bardi E.J, Langgley C.J, “The Management of Business Logistics", Thomson Asia2003.

8. DavidSimchiLevi,PhilipKamiskyAndEdithSimchi Levi,DesigningAndManagingTheSupplyChain,Ir win McGraw Hill, New York,2000.
9. Mohanty R.P And DeshmukhS.G, Advanced Operation Management, Pearson Education2003.

10. Michel Quayle \& Bryan Jones, Logistics- An Integrated Approach, Tudor Disiness Publishing,UK, 1999.

11. Raghuram G \& N Nahgaraj- Logistics And Supply Chain Management,McMillan India Ltd2001.

12. R.Sarin(Brigadier), Automating And Spare Parts Inventory Management, IndianManagement,Febuary2000.

13. Vittal N. \& B.S. Sahay, Supply Chain Management For Global Competitiveness, Macmillan-1999. 